\title{
Two adult females with a distinct familial mental retardation syndrome: non-progressive neurological symptoms with ataxia and hypotonia, similar facial appearance, hypergonadotrophic hypogonadism, and retinal dystrophy
}

Jean-Pierre Fryns, Claudia Van Lingen, Koenraad Devriendt, Eric Legius, Peter Raus

\begin{abstract}
We describe a familial mental retardation syndrome in a 30 year old, moderately mentally retarded female and her 59 year old maternal aunt. Both presented the distinct association of (1) a nonprogressive neurological syndrome with ataxia and hypotonia, (2) similar craniofacial appearance with a narrow forehead, prominent supraorbital ridges and tendency to synophrys, deeply set eyes with short, upward slanting palpebral fissures, short philtrum, and large mouth, (3) hypergonadotrophic hypogonadism, and (4) retinal dystrophy with onset in early adulthood.

(F Med Genet 1998;35:333-335)
\end{abstract}

Keywords: mental retardation; ataxia; hypergonadotrophic hypogonadism; retinal dystrophy

\section{Case reports}

The index patients are two adult females, case 1 , a 30 year old, moderately mentally retarded

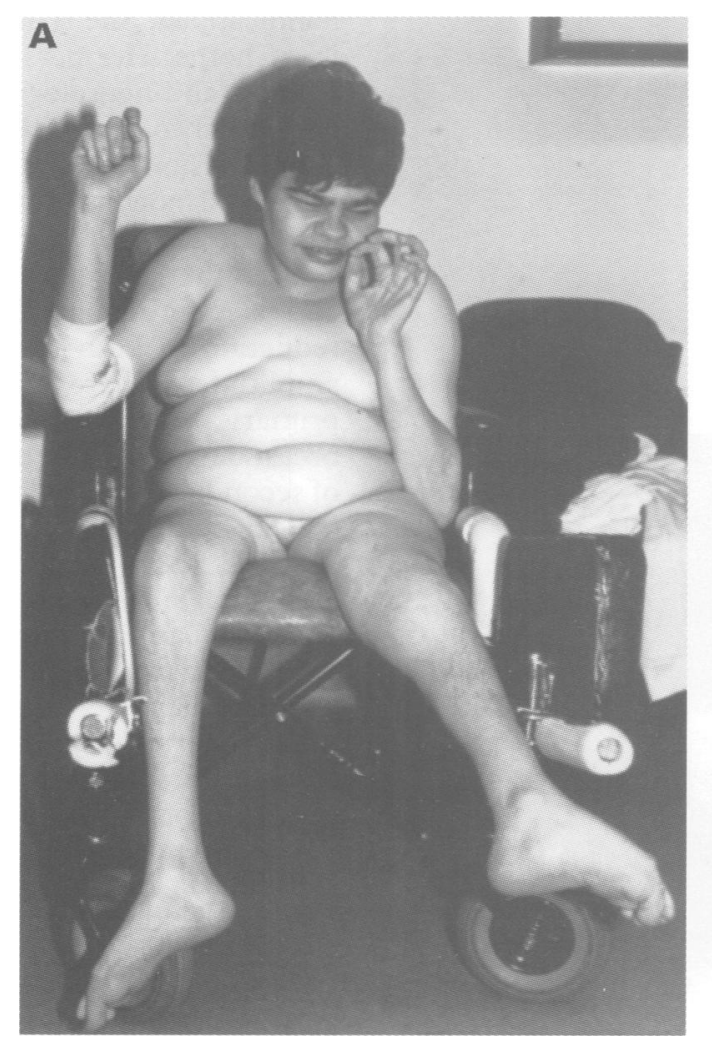

Figure 1 Similar general habitus of (A) patient 1 and (B) patient 2. woman and case 2, her maternal aunt, 59 years old and mildly mentally handicapped. Case 1 is the youngest born in a sibship of five children. Her two brothers and two sisters are normal. Case 2 is the youngest of seven children. Her oldest brother died in the perinatal period after a complicated delivery. Her other brothers and sisters, including the mother of case 1 , are normal.

Recently, the family was seen at the genetic clinic for genetic counselling and diagnosis in the two handicapped female relatives. All members of this family live in a small village, but there was no evidence of consanguinity.

\section{CASE 1}

Case 1 (figs $1 \mathrm{~A}$ and $2 \mathrm{~A}$ ), now 30 years old, was born at home after a normal pregnancy. No precise data about the perinatal and neonatal period are available, but in the first year of life no problems were noted. From the age of 1 year psychomotor retardation became evident. She has never been able to walk independently

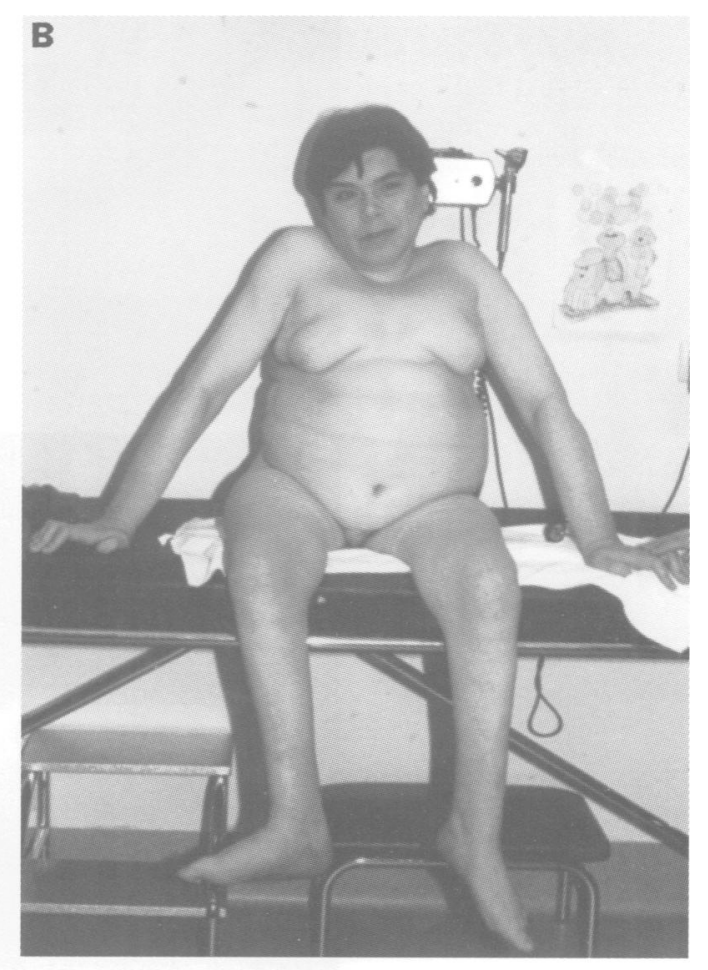

\section{Centre for Human Genetics, University Herestraat 49, B-3000 Leuven, Belgium $C$ Van Lingen}

Ophthalmologist, Mol Belgium

Correspondence to: Professor Fryns.

publication 21 October 1997




and can only sit with support. At the age of 7 years she was integrated in a school for the moderately mentally retarded. Since the age of 15 years she has been cared for at home by her older sister. She can speak a few words. Her social behaviour is friendly and she has never shown behavioural problems. At the age of 18 years she underwent an ophthalmological evaluation because poor vision had progressively been noted. Visual acuity was RE 1/10 and LE $1 / 10$. In both eyes fundoscopy showed areas of chorioretinal atrophy that were mainly localised to the posterior pole with predominant macular involvement and fine pigmentary changes, thin retinal vessels, and prominence and sclerosis of choroidal vessels. The retinal periphery showed minimal pigmentary abnormalities. On follow up examination the retinal appearance had gradually worsened. An electroretinogram at the age of 29 years showed a marked reduction in both the photopic and scotopic amplitudes.

She had no menstruation and never developed secondary sexual characteristics apart
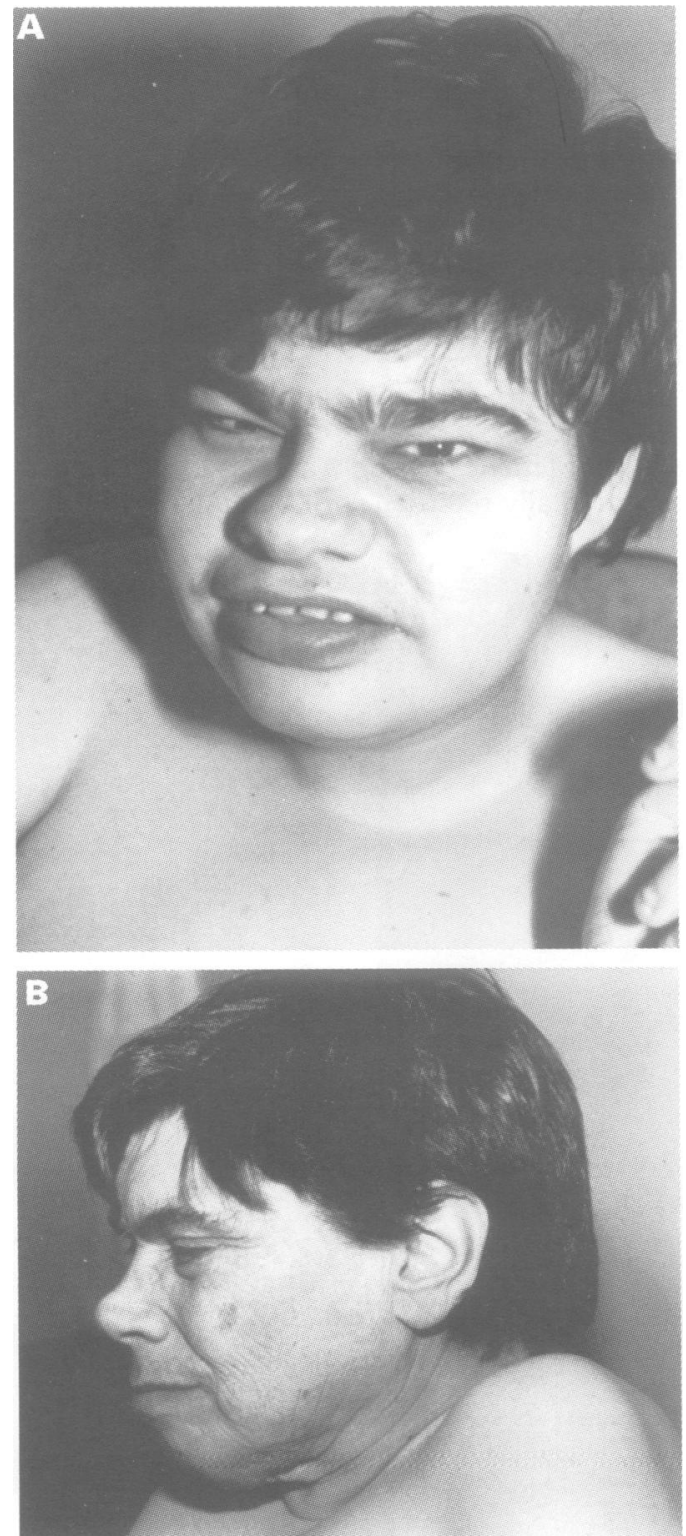

Figure 2 Same facial gestalt of $(A)$ patient 1 and $(B)$ patient 2. from minimal breast development. External genitalia were small. Endocrinological studies showed markedly raised LH (30.9 U/l) and FSH (86.1 U/l) indicating a hypergonadrophic form of hypogonadism.

Neurological examination at the age of 29 years showed marked axial hypotonia. She had never been able to sit without support and has thoracolumbar torsion scoliosis, relative truncal obesity, and hypotrophic lower legs. Ataxia was present with scanning speech, intention tremor, and marked incoordination in fingernose testing. The upper and lower limb reflexes were normal. There was bilateral pes cavus, but no other motor deficit. Sensory action potentials, motor conduction velocity, and electromyography were normal. According to the normal family members, the neurological symptoms were present from the beginning and had not changed with age. Head circumference is $55.5 \mathrm{~cm}$ and height $\pm 180 \mathrm{~cm}$. The craniofacial appearance is distinct with a narrow forehead, prominent supraorbital ridges, synophrys, deeply set eyes with short, slightly upward slanting palpebral fissures, short philtrum, large mouth with everted lower lip, and diastemata of all teeth.

Chromosome studies on a peripheral blood lymphocyte culture showed a normal $46, \mathrm{XX}$ female karyotype after $G$ and $R$ banding. Serum phytanic levels were not increased. All routine haematological, biochemical, and metabolic investigations were within the normal range. Brain neuroimaging studies could not be performed.

CASE 2

Case 2 (figs $1 \mathrm{~B}$ and 2B), the maternal aunt of case 1 , is now 59 years old and mildly mentally handicapped. She is also cared for by the family at home. Her personal history is identical. Clinical and neurological symptoms and signs are similar to the findings in case 1 .

Poor vision was first noted at the age of 20 years and now, at the age of 59 years, visual acuity is RE $1 / 20$ and LE $1 / 10$. Fundoscopy showed the same signs of chorioretinal atrophy and electroretinography showed a complete reduction in both the photopic and scotopic amplitudes. There was delayed puberty and primary amenorrhoea. Apart from scanty breast development, there was no development of secondary sexual characteristics. Endocrinological studies showed high values of LH (32.8 $\mathrm{U} / \mathrm{l})$ and FSH (92.9 U/1).

She has never been able to walk independently, but can sit without support. Neurological symptoms are identical with axial hypotonia, hypotrophic lower legs, ataxia and intentional tremor, normal upper and lower limb reflexes, marked dorsolumbar scoliosis, relative truncal obesity, and hypotrophic lower legs. Head circumference is $55 \mathrm{~cm}$ and height $170 \mathrm{~cm}$. Craniofacial dysmorphism also includes narrow forehead, prominent supraorbital ridges, synophrys, deeply set eyes with short, upward slanting palpebral fissures, short upper lip, everted lower lip, diastemata of all teeth, and a short neck. 
Chromosome studies on a peripheral blood lymphocyte culture showed a normal 46,XX female karyotype after $G$ banding. Routine haematological and biochemical investigations were normal. Brain neuroimaging studies could not be performed.

\section{Discussion}

The clinical and neurological findings in these two female relatives, a 29 year old, moderately mentally retarded female and her 59 year old, mildly mentally retarded maternal aunt, can be summarised as follows: (1) a non-progressive neurological syndrome with ataxia and hypotonia without signs of spastic paresis, (2) hypergonadotrophic hypogonadism, (3) progressive retinal dystrophy with first clinical signs in early adulthood, and (4) distinct craniofacial appearance.

The nosology and classification of the hereditary ataxias remain difficult, and some are accompanied by non-neurological manifestations, for example, cardiac involvement and endocrine dysfunction. The triad of spinocerebellar ataxia, chorioretinal dystrophy, and hypogonadotrophic hypogonadism (BoucherNeuhaüser syndrome) is an autosomal recessive disorder, reported in a few families so far. ${ }^{1-3}$ In contrast to the findings in the present two females, the neurological findings are slowly progressive and the hypogonadism is of central, hypogonadotrophic type. Recently, Rump et $a l^{4}$ reported two sibs with BoucherNeuhaüser syndrome and provided a review and analysis of previously reported cases and the differential diagnosis.

Skre et at reported two kindreds with Marinesco-Sjögren syndrome in three sibships and observed hypergonadotrophic hypogonadism in five of the six affected. The authors discussed this unusual association and commented that hypogonadotrophic hypogonadism appar- ently secondary to the neurological disorder had been documented in several heredodegenerative nervous disorders. An adequate explanation for the hypergonadotrophic hypogonadism was not available and the authors considered genetic linkage between two independent disorders as the most likely explanation for the observed concurrence.

The two adult females of this report present, in addition to the triad of ataxia, retinal dystrophy, and hypergonadotrophic hypogonadism, a distinct craniofacial appearance, which is not present in their normal relatives.

We were not able to find other reports of the concurrences of ataxia and retinal dystrophy, retinal dystrophy and hypergonadotrophic hypogonadism, or ataxia, retinal dystrophy, and hypergonadotrophic hypogonadism associated with these craniofacial features. The association of clinical and neurological symptoms in the present two female relatives may be the manifestation of another syndromic form of ataxia-hypogonadism, distinct from the Boucher-Neuhäuser syndrome. Like BoucherNeuhaüser syndrome, this triad most probably represents a hitherto unrecognised single gene disorder with an autosomal recessive pattern of inheritance.

1 Boucher BJ, Gibberd FB. Familial ataxia, hypogonadism and retinal deterioration. Acta Neurol Scand 1969;45:50710.

2 Neuhäuser G, Opitz JM. Autosomal recessive syndrome of cerebellar ataxia and hypogonadotropic hypogonadism. Clin Genet 1975;7:426-34.

3 Salvador F, García-Arumi J, Corcóstegui J, Minoves T, Tarrus $\mathrm{F}$. Ophthalmologic findings in a patient with cerebellar ataxia, hypogonadotropic hypogonadism, and chorioretinal dystrophy. Am $\mathcal{F}$ Ophthalmol 1995;120:241-4.

4 Rump P, Hamel BCJ, Pinckers AJLG, Van Dop PA. Two sibs with chorioretinal dystrophy, hypogonadotrophic hypogonadism, and cerebellar ataxia: Boucher-Neuhaüser hypogonadism, and cerebellar ataxia: Bo
syndrome. $₹$ Med Genet 1997;34:767-71.

5 Skre H, Bassöe HH, Berg K, Frövig AG. Cerebellar ataxia and hypergonadotropic hypogonadism in two kindreds. Chance concurrence, pleiotropism or linkage? Clin Genet 1976;9:234-44. 